\title{
La teoría del valor trabajo: un diálogo enmascarado en el intercambio de heridas
}

\author{
El único invariante consistente, tanto lógica como \\ conceptualmente, es la propia definición de valor agregado \\ de la teoría del valor-trabajo, ya que ningún otro invariante es \\ consistente en el caso general con esta definición, que constituye \\ la base de la teoría, y con el resto de sus proposiciones inherentes.
}

Homero Cuevas

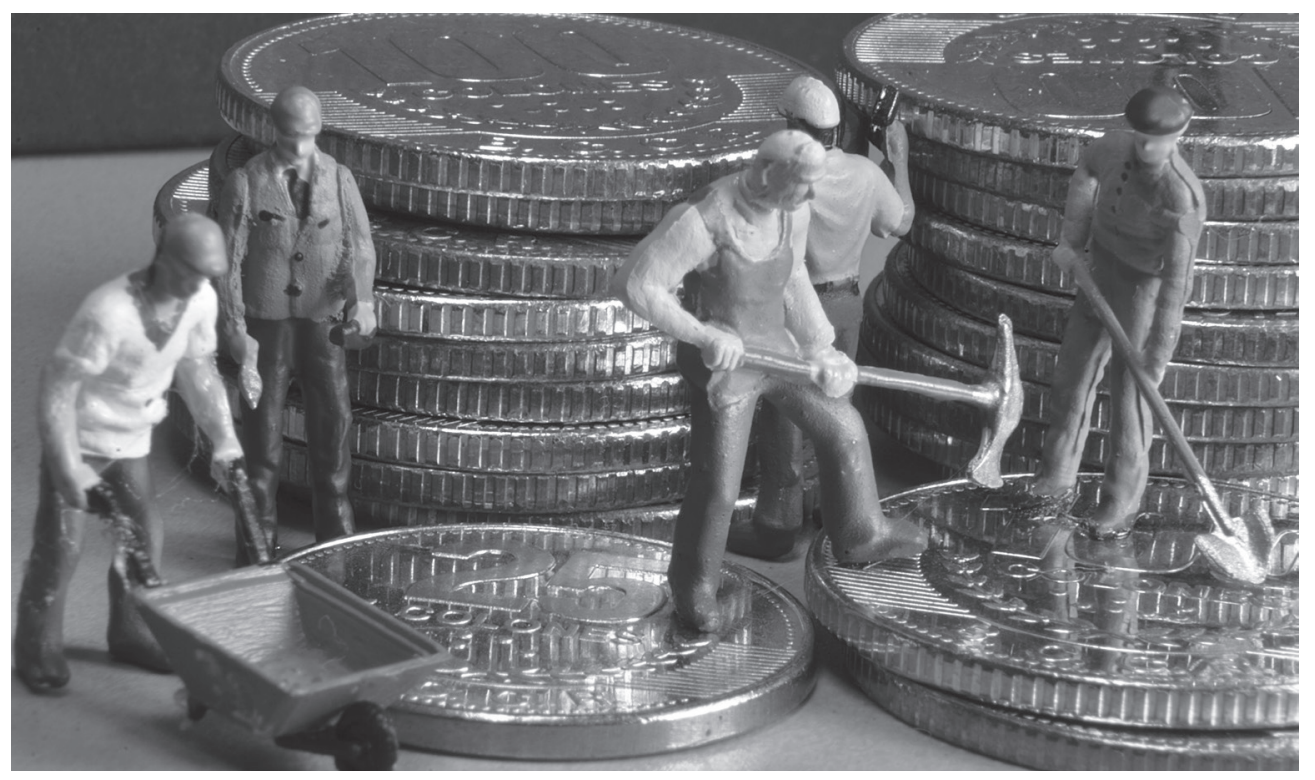

Todos los días intercambiamos horas gastadas de nuestra jornada, aunque la piel del encuentro solo sea el dinero, la mayoría de nosotros entrega su trabajo al otro tatuado en monedas, casi siempre anónimo, a cambio de objetos y servicios (suponemos el trabajo del otro), ofrecidos como mercancías. Siglos atrás ese intercambio se hacía entre productores directos, a duras penas se usaba el dinero para valuar productos disímiles entre personas cercanas; aunque siempre quedara la duda sobre pequeñas diferencias en el intercambio de heridas.

La complejidad de la evolución del intercambio fue llevando a desentrañar las valuaciones, especialmente en dinero y en tiempo de trabajo, para dejar de lado todos los demás factores (no económicos) que intervienen en las relaciones de producción, en particular, la relaciones personales entre productores y consumidores. En la medida en que se desarrollaron el capitalismo y la modernidad se hicieron complejas las relaciones económicas y de racionalización de la vida, entonces las relaciones sociales han sufrido un alto grado de despersonalización. 
No solo se ha hecho complejo el intercambio, también el entramado de las relaciones, pero sobre todo, las valoraciones ideológicas (religiosas, de solidaridad, culturales, etc.) se han diluido en el ámbito privado. Y dada la complejidad del sistema productivo y la distancia sobre la génesis de la construcción de valor en los productos debido a que la información ha quedado reducida a los precios, ni los individuos ni las empresas tienen posibilidades de comparar ni las valoraciones del tiempo trabajado ni el valor-trabajo de los precios del intercambio.

Toda persona es rica o pobre según el grado en que pueda disfrutar de las cosas necesarias, convenientes y agradables de la vida. Pero una vez que la división del trabajo se ha consolidado, el propio trabajo de cada hombre no podrá proporcionarle más que una proporción insignificante de esas tres cosas. La mayoría de ellas deberá obtenerlas del trabajo de otros hombres, y será por tanto rico o pobre según sea la cantidad de ese trabajo de que pueda disponer o que sea capaz de comprar. Por lo tanto, el valor de cualquier mercancía, para la persona que la posee y que no pretende usarla o consumirla sino intercambiarla por otras, es igual a la cantidad de trabajo que le permite a la persona comprar u ordenar. El trabajo es, así, la medida real del valor de cambio de todas las mercancías (Smith, 1996, p. 64).

Estas circunstancias de la modernidad capitalista ensombrecen los orígenes y posibles soluciones de la mayor injusticia socioeconómica contemporánea: la desigualdad económica en sus principales dimensiones como son el ingreso y la propiedad. Esto ha alimentado, desde un clásico como Smith, la necesidad de una medida de valor invariable, un lenguaje común para el intercambio:

Pero aunque el trabajo es la medida real del valor de cambio de todas las mercancías, no es la medida con la cual su valor es habitualmente estimado. Es con frecuencia difícil discernir la proporción entre dos cantidades distintas de trabajo. El tiempo invertido en dos tipos diferentes de labor no siempre bastará por sí solo para determinar esa proporción. Habrá que tener en cuenta también los diversos grados de esfuerzo soportado y destreza desplegada. Puede que haya más trabajo en una hora de dura labor que en dos de una tarea sencilla; o en una hora de un oficio cuyo aprendizaje costó diez años que en un mes de un trabajo común y corriente. Pero no es fácil encontrar una medida precisa ni de la fatiga ni de la destreza (Smith, 1996, pp. 65-66).

Y esto por supuesto lleva a desvirtuar y confundir sobre los méritos y valoraciones de los trabajadores respecto a su aporte a los procesos productivos y sobre el modo como se asume la distribución del producto. Cada día se oculta más en el intercambio de nuestras manos el rostro de nuestras horas trabajadas, el pasado incierto de nuestros o vuestros antepasados, o ya del robo de alguno, deciden sobre el poder de comprar entre nosotros. El rastreo histórico, jurídico y económico de la propiedad, requiere un modo de comparación transversal (tiempo) y vertical (productos). Ante esta necesidad Smith lanza un ancla a las turbulentas aguas del intercambio de las horas trabajadas por los individuos, en la puesta en escena de sus productos objetivados: el mercado. 
Es evidente, por lo tanto, que el trabajo es la única medida universal y precisa del valor, o el único patrón mediante el cual podemos comparar los valores de distintas mercancías en cualquier tiempo y lugar. No podemos estimar, por supuesto, el valor real de diversas mercancías de un siglo a otro según las cantidades de plata que se entregan a cambio de ellas. Tampoco podemos estimarlo de un año a otro según las cantidades de cereal. Pero según las cantidades de trabajo podemos estimarlo con la mayor precisión tanto de un siglo a otro como de un año a otro (Smith, 1996, pp. 72-73).

El intercambio medido en precios oculta no solo su valor, enmascara también la plusvalía; transforma el modo de relacionarnos en la sociedad, traslada el sacrificio del tiempo entregado en el intercambio (disuelve la polis), nos atrapa en una complejidad institucionalizada en la que la alienación de los individuos va tomando la forma del capital: las horas no consumidas se apilan en los leños secos ya cortados, a salvo, que aseguran el fuego del mañana. Pero las horas contadas no son suficientes para la contabilidad del intercambio. Los sueños y los deseos no compartidos obligan al sacrificio desde las circunstancias de cada ruta en el calendario personal. El precio de mercado mide entonces, más allá del trabajo incorporado, los apremios históricos de la entrega de los participantes desde cada orilla. Entonces la justicia del tiempo en el intercambio multiplica sus rostros hasta perdernos en sus laberintos: nos abruma el salario tasado bajo la historia de nuestras manos arbitrada por el capital. $\mathrm{Y}$ en este camino revisitado de los clásicos de la economía acudimos a Ricardo para comentar cómo este lenguaje del precio teje desviaciones del precio natural, de las horas de intercambio:

Al considerar al trabajo como base de los bienes, y la cantidad comparativa de trabajo que es necesaria para su producción, como la regla que determina las cantidades que deben entregarse a cambio de cada uno de los otros, no debemos suponer que negamos las desviaciones accidentales y temporales que registran los precios reales o de mercado de los bienes, en relación con su precio primario y natural (Ricardo, 1997, p. 67).

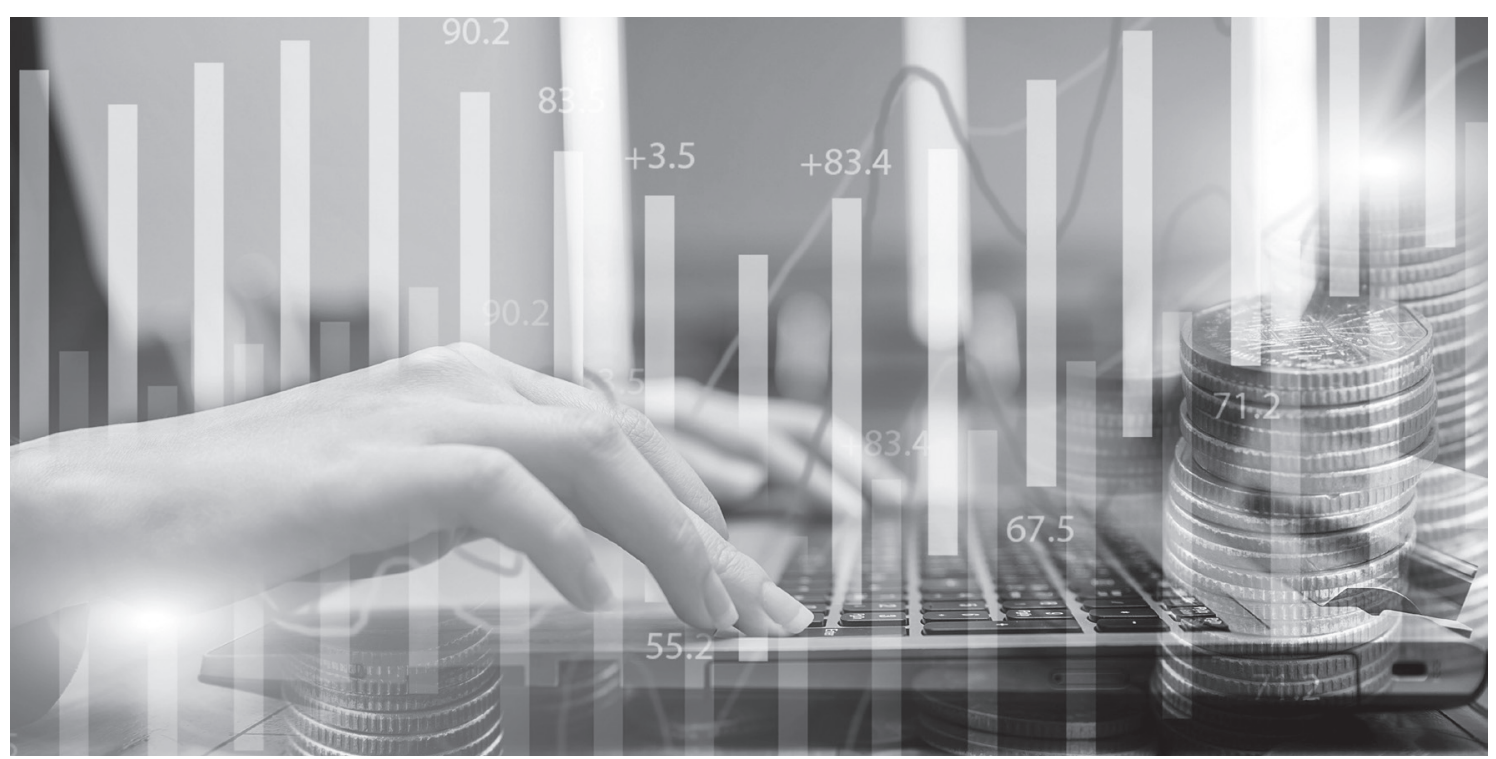


Entonces, la necesidad de encontrar un invariante es no solo apremiante, sino una condición para construir el diálogo por lo justo; el imperativo de esta búsqueda no puede abolir las dificultades para encontrarlo. Y por definición, por la naturaleza del fenómeno, solo el valor-trabajo puede dar cuenta de una teoría del valor camino de la justicia; otras valoraciones serán eficientes, o medirán un horizonte de la historia; pero el invariante del trabajo no solo se ocupa del intercambio sino de la historia del lenguaje usado como moneda, y esa lengua funda realidades. La cantidad de trabajo intercambiada fija fonemas, sonidos universales. Entonces el problema ya no estará en las palabras como ropaje de las mercancías, sino en la tragedia individual de cada uno para establecer las fronteras de la mercancía en su ser. Y siguiendo a Marx, la fuerza de trabajo como mercancía es el origen y el fin de la pregunta: "El proceso de consumo de la fuerza de trabajo es, al mismo tiempo, el proceso de producción de la mercancía y de la plusvalía" (Marx, 1954, p. 485).

Y, la unidad de medida es medida por ella misma. Esta trampa del lenguaje, ya estudiada en otras esferas del conocimiento (Wittgenstein), hace de este debate económico un hecho estructurante-estructurado (Bourdieu):

El valor de la fuerza de trabajo, como el de toda otra mercancía, lo determina el tiempo de trabajo necesario para la producción, incluyendo, por tanto, la reproducción de este artículo específico. Considerada como valor, la fuerza de trabajo no representa más que una determinada cantidad de trabajo social medio materializado en ella. La fuerza de trabajo sólo existe como actitud del ser viviente. Su producción presupone, por tanto, la existencia de este (Marx, 1954, p. 475).

En este pequeño breviario sobre la pregunta del valor-trabajo he elegido de un modo caprichoso, tal vez personal, las huellas más evidentes legadas por el profesor Homero Cuevas alrededor de los enigmas de esta pregunta por el fantasma de la transformación de los valores a precios, de esta contabilidad entre el tiempo de trabajo y los precios del intercambio. Y nos ocupa este tema de modo central en esta entrega de la revista Papeles, porque...

La teoría económica debe ser realista, en el sentido de que debe ser dirigida al análisis de la economía capitalista en la que la propiedad del capital da poder, además de renta, y donde las decisiones de los trabajadores, consumidores y hogares tienen una importancia muy limitada (King, 2009, p. 59).

Entonces, el núcleo relevante de la pregunta, tanto en el sentido de la construcción teórica del problema, como en el sentido de la pregunta esencial por una ciencia económica que dé cuenta de un mundo más justo, se concentra en torno al problema de los valores y precios de los salarios (quedan aplazados dos interrogantes fundantes: el salario justo y el modo como se puede llegar de modo justo al salario ${ }^{1}$ ). El profesor Homero Cuevas dedicó buena parte de su vida académica a la pregunta por el valor

1 "El valor de la fuerza de trabajo se reduce al valor de una determinada suma de medios de vida. Cambia, por tanto, al cambiar el valor de éstos, es decir, al aumentar o disminuir el tiempo de trabajo necesario para su producción" (Marx, 1954, p. 479). 
abstracto de los salarios como único invariante coherente: "Todo lo que se requiere para una correcta determinación de todos los precios y la tasa de ganancia es el valor abstracto de los salarios" (Cuevas, 1984, p. 12).

El desarrollo de la teoría del valor en sus laberintos invita a una mirada holística, macroeconómica: "En términos de la teoría del valor trabajo, el valor agregado en la economía, no es otra cosa que el trabajo agregado a los medios de producción durante el proceso productivo" (Cuevas, 1984, p. 15). Esta es la razón por la que la mejor opción como medida de todas las mercancías no puede ser otro que el trabajo. Y los caminos de este invariante deben suponer dos perspectivas: 1) el trabajo es el único invariante posible coherente (por definición); y 2) solamente una solución global de los sectores económicos puede dar cuenta del problema entre valores y precios (los postulados microeconómicos no siempre se cumplen en los agregados macroeconómicos ${ }^{2}$ ). Un resumen del proceso crítico sobre el problema del valor abstracto del trabajo es:

Samuelson (1971) encontró que solamente cuando cada sector de la economía satisface las condiciones de una mercancía patrón Sraffiana se obtienen simultáneamente la igualdad de suma de precios de producción a suma de valores y de suma de ganancias a suma de plusvalías. Más tarde, Morishima (1973) demostró que la condición requerida era algo menos restrictiva: la economía como un todo debería satisfacer las condiciones de una mercancía patrón Sraffiana, lo cual no implica que necesariamente cada sector individual deba satisfacerlas. Pero, en cualquier caso, como concluye Samuelson, tales condiciones están demasiado distantes de la realidad para pretender que un conjunto de proposiciones restringidas exclusivamente a ellas puedan ser presentadas como una teoría económica relevante (Cuevas, 1984, p. 15).

Y aunque...

el salario fluctúa de acuerdo con las más diversas circunstancias, como la escasez o abundancia relativa de brazos frente a la acumulación de capital, lo cual lo hace tan inadecuado como patrón de medida como el resto de las mercancías ordinarias, "iguales cantidades de trabajo, en todos los tiempos y lugares, tienen el mismo valor para el trabajador" (Cuevas, 1986, p. 14).

El precio de la cantidad de trabajo en el intercambio puede vestirse con máscaras, con valoraciones previas, con los privilegios de las asignaciones iniciales; sin velo de la ignorancia ni posición original (Rawls); pero las horas gastadas de cada uno de nosotros, esas horas que le arrancamos a la cita ineludible con la muerte, son una medida de intercambio invariable como valor en el tiempo, aunque su precio

2 The aggregate production function is a fundamental neoclassical construct. At the theoretical level, it is used in virtually every branch of economics analysis. At the empirical level, it is used to analyze the determinants of technical change and capacity utilization, and almost half a century after Solow's celebrated 1957 article, it remains the method of accounting for the determinants of growth. Yet the theoretical foundations of this construct are shaky, because it cannot be grounded in any plausible micro-foundation [...] It is curious that a tradition so insistent on the necessity of micro-foundations should rely so heavily on a construction that cannot be derived from micro-foundations (Shaikh, A., 2005, p. 447) 
distorsione el intercambio de esfuerzos, aunque el precio transforme el tiempo en un inventario adquisitivo desigual.

Hoy, intercambiamos precios, objetos sin rostro, horas sin historia. Vivimos en un mundo de gran inestabilidad en las valoraciones económica, moral y biológica; retomar el estudio de la teoría del valor trabajo podría trazar rutas para un mundo más libre y más equitativo: más justo.

Y como dijera el maestro Homero Cuevas en su libro La teoría del valor-trabajo y el sistema de precios (1986), para cerrar su libro (parafraseo de memoria): no sabemos cuál es la teoría del valor correcta con certeza, pero estamos seguros que es necesario tener una teoría del valor en economía, a lo mejor, la suma de todas la teorías. Esta entrega de la revista Papeles invita a reflexionar desde este tema la problemática socioeconómica de hoy, en Colombia y en el mundo.

Enrique Ferrer-Corredor

\section{Referencias}

Cuevas, H. (1984). La transformación correcta. Cuadernos de Economía, 6 (7), pp. 9-34. Bogotá: UNAL.

Cuevas, H. (1986). Valor y sistema de precios. Bogotá: UNAL.

King, J. E. (2009). Una historia de la economía poskeynesiana desde 1936. Madrid: Akal.

Marx, C. (1954). El capital. Madrid: Luarna ediciones.

Ricardo, D. (1997). Principios de Economía politica y tributación. México: FCE.

Shaikh, A. (2005). Nonlinear Dynamics and Pseudo-Production Functions. Eastern Economic Journal, 31, (3, Summer), pp. 447-466. London: Palgrave Macmillan Journal.

Smith, A. (1996). La riqueza de las naciones. Madrid: Alianza Editorial. 